\title{
Algunas claves para escribir correctamente un artículo científico
}

\author{
ANDREA VILLAGRÁN T. ${ }^{1}$, PAUL R. HARRIS D. ${ }^{1}$ \\ 1. Departamento de Pediatría, Unidad de Gastroenterología y Nutrición Pediátrica, Facultad de Medicina, \\ Pontificia Universidad Católica de Chile, Santiago, Chile.
}

\begin{abstract}
\section{Some key factors in medical writing}

The purpose of scientific research is to disseminate their results in a publication. Scientists, clinicians, graduate and postgraduate students are judged and are primarily known for their publications. A scientific experiment whilst their results may be outstanding does not end until those results are published. Thus, the scientists not only have to "do" science but also "write" science. A poor wording could prevent or delay the publication of an excellent scientific work. The purpose of this article is to help scientists and students to prepare manuscripts that are likely to be accepted for publication and to be perfectly understood when issued. As the requirements journal vary widely among disciplines, and even within a single discipline, it is not possible to make recommendations that are universally acceptable. Therefore, this article will get its importance, insofar as it is taken as a guide for those professionals who wish to publish their studies in national and international journals.

(Key words: scientific article, redaction, manuscript).

Rev Chil Pediatr 2009; 80 (1): 70-78
\end{abstract}

\section{RESUMEN}

El objetivo de la investigación científica es divulgar sus resultados en una publicación. Hombres y mujeres de ciencia, médicos clínicos, estudiantes graduados y de postgrado son juzgados principalmente y se los conoce por sus publicaciones. Un experimento científico, por espectaculares que sean sus resultados, no termina hasta que esos resultados se publican. Así pues, el científico no sólo tiene que "hacer" ciencia sino también "escribir" ciencia. Una mala redacción puede impedir o retrasar la publicación de un trabajo excelente. El propósito de este artículo es ayudar a científicos y estudiantes a preparar manuscritos que tengan posibilidades de ser aceptado para su publicación y de ser perfectamente entendidos cuando se publiquen. Como los requisitos exigidos por las revistas varían mucho según las disciplinas, e incluso dentro de una misma disciplina, no es posible hacer recomendaciones que sean universalmente aceptables. Por lo tanto, el presente artículo obtendrá su importancia, en la medida que sea tomado como una guía por aquellos profesionales que deseen publicar sus estudios en diferentes revistas nacionales e internacionales.

(Palabras clave: artículo científico, redacción, manuscritos).

Rev Chil Pediatr 2009; 80 (1): 70-78

Trabajo recibido el 04 de diciembre de 2008, aceptado para publicación el 26 de enero de 2009.

Correspondencia a:

Dr. Paul R. Harris D.

E-mail: pharris@med.puc.cl 


\section{Introducción}

Uno de los grandes problemas que enfrenta un investigador surge cuando tiene que dar a conocer sus resultados, pues escribir no resulta tarea fácil, más aún cuando el mundo actual se mueve en una especie de perfeccionamiento del vocabulario científico y surgen palabras muy complejas, como manifestación de dichos cambios. A partir de esta problemática, el presente trabajo tiene el objetivo de proponer un grupo de sugerencias que contribuyan a que la labor de redactar un artículo científico se convierta en un acto de placer y de disfrute por parte de quién tiene la difícil misión de buscar soluciones a los problemas y que sean conocidos por todos: comunidad científica, profesionales y la sociedad en general.

\section{Un poco de historia}

El primer libro que se conoce, inscrito en una tablilla de arcilla de alrededor del 4000 a.C., anterior al génesis en unos 2000 años, es un relato caldeo del diluvio ${ }^{1}$. El primer medio utilizado en forma masiva fue el papiro alrededor del año 2000 a.C., luego en el año 190 a.C., se utilizó el pergamino. En el año 105 de nuestra era los chinos inventaron el papel, luego vino la imprenta de Gutenberg en el año 1455 con lo cual se imprimió la primera biblia de 42 renglones. Las primeras bibliotecas fueron hechas por los griegos, 50 años a.C. en Efeso y Pergamo (hoy Turquía) ${ }^{2}$.

Los seres humanos han sido capaces de comunicarse desde hace milenios. Sin embargo, la comunicación científica, tal como hoy la conocemos, es relativamente nueva. Las primeras revistas científicas se publicaron sólo hace 300 años, en 1665 la Journal des Sçavans en Francia y las Philosophical transactions of the Royal Society of London en Inglaterra. Más actual aún, la clásica organización del artículo científico llamada IMRYD (introducción, métodos, resultados y discusión) se ha creado en los últimos 100 años.

Las primeras revistas publicaban artículos que llamamos "descriptivos". De forma típica, un científico informaba: "primero vi esto y lue- go vi aquello", o bien: "primero hice esto y luego hice aquello". A menudo las observaciones guardaban un simple orden cronológico. Este estilo descriptivo resultaba apropiado para la clase de ciencia sobre la que se escribía. De hecho, ese estilo directo de informar se emplea aún hoy en las revistas a base de "cartas al editor" o en los informes médicos sobre casos clínicos $^{2}$.

\section{La publicación científica es la etapa final de un proceso}

La ciencia moderna es muy distinta de la ciencia del pasado. En nuestros días la ciencia se ha institucionalizado y es una profesión más, con sus propias normas de acceso y sus reglas implícitas y explícitas. Hace tiempo que los científicos dejaron de trabajar únicamente por el amor al conocimiento, aunque no cabe duda de que ésta sigue siendo todavía una de sus motivaciones más poderosas. Puede afirmarse pues, que una parte del contrato implícito del científico con la sociedad consiste precisamente en el intercambio de productos mentales y resultados útiles para la comunidad con reconocimiento público por parte de sus colegas ${ }^{3}$. ¿Cómo se expresa este reconocimiento?. De muy variadas maneras, sin embargo, la más reconocida y considerada por muchos como de mayor apoyo social, es la publicación en revistas académicas de alto prestigio. Por ello las principales motivaciones que llevan a un científico a publicar sus experiencias y resultados son:

1. Para establecer una carrera académica. A los hombres de ciencia se les conoce (o no se les conoce) por sus publicaciones.

2. Para el currículum del investigador en busca de trabajo.

3. Intercambio de resultados de investigación y colaboración internacional.

4. Una obligación impostergable. Propio de las carreras y profesiones científicas; lo que no está escrito... no está hecho. Un experimento o un trabajo científico por espectaculares que sean sus resultados, no termina hasta que se publica.

5. La piedra angular en ciencia es que las 
investigaciones originales tienen que publicarse:

- Para verificarse (reproducibilidad).

- Para aceptarse e ingresar al "conocimiento científico".

- No sólo hay que hacer ciencia, sino escribir ciencia.

\section{¿Qué es un artículo científico?}

El científico siempre ha buscado cómo dar a conocer sus pensamientos. En el siglo XVII éstos intercambiaban sus ideas por medio de cartas; siendo este un método limitado pues no permitía conocer las diferentes posiciones que existían acerca de un fenómeno científico en estudio.

En la guía para la redacción de artículos científicos publicados por la $\mathrm{UNESCO}^{4,5}$, se señala que la finalidad esencial de un artículo científico es comunicar los resultados de investigaciones, ideas y debates de una manera clara, concisa y fidedigna. "El hombre de ciencia parece ser el único que tiene hoy algo que decir, y el único que no sabe como decirlo" (Sir James Barrie). Escribir un artículo científico no significa tener dones especiales, sino requiere de destrezas y habilidades creativas que puede aprender cualquier investigador.

\section{¿Cómo escribir un artículo científico?}

Inicialmente las publicaciones eran descriptivas (como las cartas actuales), pero a mediados del siglo XIX, gracias a Pasteur y Koch, que confirmaron la teoría microbiológica de las enfermedades, se hizo necesario describir en forma detallada la metodología para acallar a los fanáticos de la generación espontánea y el dogma de la reproducibilidad se hizo central. Esto fue el principio del IMRYD. Luego vino el desarrollo de la microbiología, avances milagrosos y se crearon muchos fondos de apoyo a la investigación, esto creó ciencia y la ciencia produjo artículos, de modo que las revistas debieron exigir cada vez más publicaciones precisas, sucintas, por problemas de espacio físico gatillando la maduración del IMRYD. El formato IMRYD no es más que un sistema para organizar un trabajo científico, y consiste en responder 4 preguntas claves (figura 1).

\section{Estructura de un artículo científico}

Título. Es relevante como una guía para el que lee o busca un trabajo. Su extensión debe tener la menor cantidad de palabras posibles que describan los contenidos del trabajo (10-12 palabras), con efectividad en la sintaxis y sin requerimientos gramaticales fuertes. Es recomendable definir un título tentativo previo a redactar el manuscrito y elaborar el título final luego de terminar el artículo. Evitar en lo posible subtítulos y abreviaciones y eliminar palabras que no informan (ej. Informe preliminar, observaciones sobre..., estudio de..., contribución a..., algunos aspectos interesantes sobre el conocimiento de...). Se recomiendan 4 opciones para un buen título (figura 2).

Resumen (abstract). Jane Rusel1 ${ }^{6}$, explica que un resumen es la representación abreviada y correcta del contenido de un documento, de preferencia preparado por el autor para publicarse junto con el documento. El propósito del

\begin{tabular}{|ll|}
\hline Introducción & $\longrightarrow$ iCuál es el problema? \\
Material y métodos & iCómo se estudió el \\
& problema? \\
Resultados & ¿Qué se encontró? \\
Discusión & iQué significan dichos \\
& hallazgos? \\
\hline
\end{tabular}

Figura 1. El formato IMRYD no es más que un sistema para organizar un trabajo científico, y consiste en responder 4 preguntas claves.

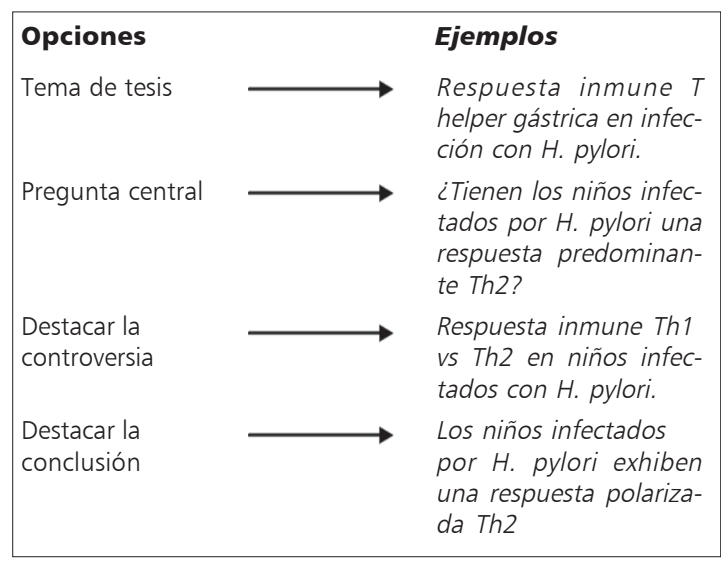

Figura 2. Se recomiendan 4 opciones para escoger un buen título. 
resumen es despertar el interés del lector por la lectura total del artículo. Generalmente, el lector lee el título, si este es interesante lee el resumen, si este es bueno, el lector continuará la lectura del artículo. Con el resumen, el título ayuda a aquellos interesados por el tema a decidir si les conviene leer el artículo o no. Es importante enfatizar que el resumen se debe entender por sí solo sin necesidad de leer el artículo ya que puede actuar como sustituto del texto si no se dispusiera de él ${ }^{7}$. Para que el lector tenga más elementos, a continuación se enumeran algunas consideraciones generales para la presentación de resúmenes:

- Mini versión del trabajo.

- La extensión máxima habitual es de 250 palabras.

- Se mantiene el mismo estilo que se empleó en el resto del trabajo.

- No se debe incluir información que no esté descrita en el artículo.

- Objetivo y enfoque del trabajo.

- Descripción de la metodología.

- Resumen de los resultados.

- Principales conclusiones.

- No se incluyen referencias.

- Debe ser escrito en pasado.

- Debe ser escrito al terminar todo el artículo.

Introducción ("lo que mal empieza, mal acaba”, Eurípides). La introducción debe responder a la pregunta de ¿porqué se ha hecho éste trabajo?. Describe el interés que el artículo tiene en el contexto científico del momento, los trabajos previos que se han hecho sobre el tema y qué aspectos son controversiales. Con la abundancia de trabajos de revisión existentes actualmente, la introducción no necesariamente debe ser muy extensa y puede beneficiarse de lo expuesto en la revisión más reciente sobre el tema.

El objetivo de este apartado es motivar al lector para que lea todo el trabajo. Centrarlo en el foco principal del trabajo, donde las referencias son claves y deben ser bien seleccionadas. Al final de la introducción el lector debería ya saber porque hicieron el estudio. La introducción generalmente termina con la presentación de la hipótesis y/o objetivos. Existen diferentes criterios sobre la organización de la introduc-

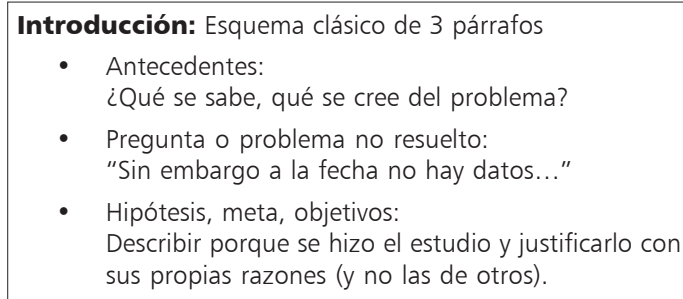

Figura 3. Existen diferentes criterios sobre la organización de la introducción y que el investigador puede tener en cuenta en el momento de redactarlo. La figura muestra el esquema clásico de 3 párrafos.

ción y que el investigador puede tener en cuenta en el momento de redactarlo (figura 3). En cualquier caso, la introducción debe ser breve, concisa y escrita en presente.

Materiales y Métodos ("la preparación de un artículo científico tiene menos que ver con el talento literario que con la organización", Robert A. Day). En esta sección se debe responder a la siguiente pregunta: ¿cómo se hizo?. Se debe dar detalle de todos y cada uno de los pasos que se siguieron para obtener los resultados, y de los materiales usados. De la Torre ${ }^{8}$, define al método como el modo de proceder o el procedimiento seguido en las ciencias para hallar la verdad y demostrarla; constituyendo así el diseño de la investigación. La metodología debe ser reproducible, de ahí la importancia de la claridad con que se exponga. Si el método es conocido sólo se menciona y se precisa la cita bibliográfica. Si es nuevo o si es un método conocido pero que se ha modificado, debe explicarse detalladamente.

La sección de materiales y métodos se puede organizar en 5 áreas:

1. Diseño: se describe el diseño del experimento (aleatorio, controlado, casos y controles, ensayo clínico, prospectivo, etc.).

2. Población: sobre la que se ha hecho el estudio. Describe el marco de la muestra y cómo se ha hecho su selección.

3. Entorno: indica dónde se ha hecho el estudio (hospital, asistencia primaria, escuela, etc).

4. Intervenciones: se describen las técnicas, tratamientos (usar nombres genéricos siempre), mediciones y unidades, pruebas piloto, aparatos y tecnología, etc. 
5. Análisis estadístico: señala los métodos estadísticos utilizados y cómo se han analizados los datos.

La figura 4 resume algunos puntos que se deben considerar y aclarar al escribir esta sección de materiales y métodos. Esta sección debe ser escrita en pasado.

Resultados ("el necio colecciona hechos, el sabio los selecciona”, John Wesley Powell). En esta sección se reportan los nuevos conocimientos, es decir, lo que se encontró y debiera ser la sección más simple de redactar. Incluye las tablas y figuras que, por sí solas, deben poder expresar claramente los resultados del estudio. Todas las tablas y figuras se citarán en el texto del artículo, comentando los datos más relevantes, de manera que sea posible comprender lo más importante de los resultados, sin que sea imprescindible consultarlo y evitando la redundancia ${ }^{7}$. Algunas consideraciones al momento de presentar los resultados:

- Estar seguro de haber entendido los resultados.

- Presentar los datos sin interpretarlos.

- Usar subtítulos para párrafos.

- Las determinaciones (y estadísticas) deben ser significativas.

- Usar sistemas internacionales de unidades, (http://www.metric1.org/).

- Usar comas (español) o puntos (inglés) para decimales.

- Obviar información innecesaria y repetitiva.

Es recomendable evitar la verborrea y no describir los métodos de nuevo. Aunque esta sección sea la más importante, generalmente es la más corta. Se debe decidir como se van a presentar los resultados (texto, tablas y/o figuras) y luego elaborar un esqueleto de la secuencia de resultados a mostrar, con los detalles en las figuras y las generalidades en el texto. Cuando la información se pueda presentar en el texto debe hacerse así. Una figura incluye todo tipo de material no tabular (morfología, algoritmos, histogramas, gráficas, fotografías, etc). La tabla tiene la ventaja de mostrar mejor los valores numéricos exactos con sus posibles interrelaciones, mientras que un gráfico expre-

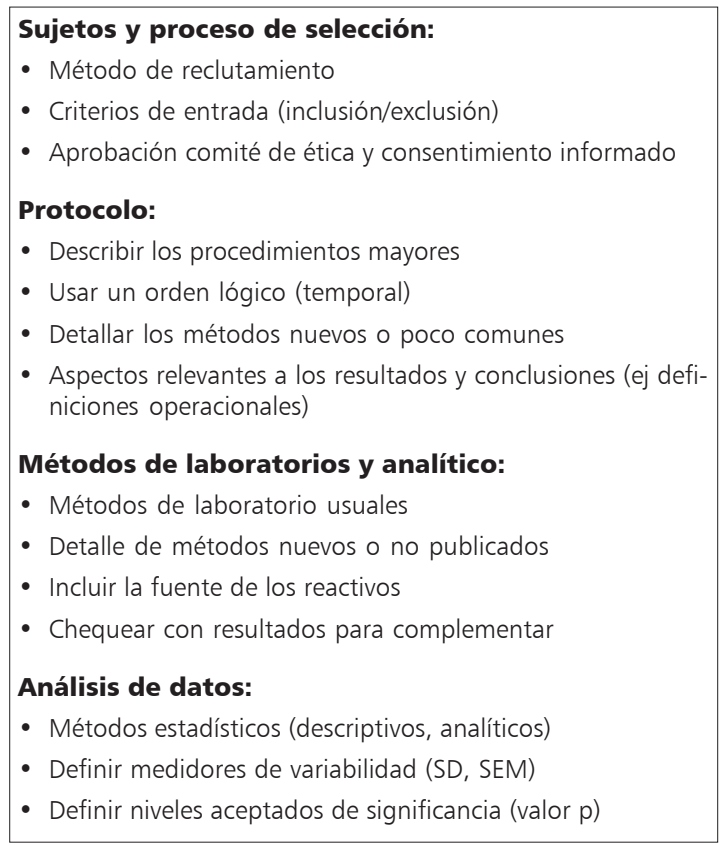

Figura 4. Puntos a considerar y aclarar al escribir la sección de materiales y métodos.

sa mejor la tendencia de los datos o patrones bien definidos. Por tanto, la tabla se utilizará cuando la precisión de los datos es importante y el gráfico cuando los datos presentan una tendencia definida o permiten resaltar una diferen$\mathrm{cia}^{7}$. Los resultados deben poder ser vistos y entendidos de forma rápida y clara. Es por ello que la construcción de ésta sección debe comenzar por la elaboración de las tablas y figuras y, sólo posteriormente, redactar el texto pertinente en función de ellas. Esta sección se debe escribir en pasado.

Discusión ("si usted va a describir la verdad deje la elegancia al sastre”, Albert Einstein). Aquella investigación que ofrezca unos buenos resultados y una buena discusión se asegura su publicación. Lo contrario ocurrirá con aquella que tenga unos buenos resultados y una mala discusión. Por eso, muchos afirman que la discusión es el corazón del manuscrito, donde la mayoría de los lectores irán después de leer el resumen y es la sección más compleja de elaborar y organizar, donde se pone a prueba la fortaleza científica de un investigador. 
En esta sección se interpretan los datos en relación a los objetivos originales e hipótesis y al estado de conocimiento actual del tema en estudio. Algunas sugerencias que nos pueden ayudar:

- Comparar conclusiones propias con la de otros autores.

- Identificar errores metodológicos.

- Alcanzar ciertas conclusiones... ¿qué es lo nuevo?.

- No repetir la presentación de resultados en forma más general.

- Escribir esta sección en presente ("estos datos indican que"), porque los hallazgos del trabajo se consideran ya evidencia científica.

- Identificar necesidades futuras de investigación (perspectivas).

- Especular y teorizar con imaginación y lógica sobre los aspectos más generales de las conclusiones. Esto puede avivar el interés de los lectores.

- Sacar a la luz y comentar claramente, en lugar de ocultarlos, los resultados anómalos, dándoles una explicación lo más coherente posible o simplemente diciendo que esto es lo que se ha encontrado, aunque por el momento no se vea explicación. Si no lo hace el autor, a buen seguro lo hará el editor o el revisor.

Se debe tener en consideración que una pobre discusión genera que el significado de los datos se oscurezca y que el artículo sea rechazado, aún teniendo datos sólidos. Además se debe estar atento a que el exceso de palabras no esté ocultando resultados o conclusiones.

Literatura citada ("los textos que contienen innumerables referencias revelan más inseguridad que erudición", William C. Roberts). Las referencias cumplen dos funciones esenciales: testificar y autentificar los datos no originales del trabajo y proveer al lector de bibliografía referente al tema en cuestión. Las revistas varían mucho en la forma de tratar las referencias. Una autora que examinó 52 revistas científicas encontró 33 estilos diferentes de enumerar las referencias ${ }^{9}$. Aunque hay una variedad casi infinita de estilos de referencias, la mayoría de las revistas científicas utilizan alguno de estos tres sistemas generales: nombre y año, numérico-alfabético y de orden de mención.

Sistema de nombre y año. Llamado a menudo sistema Harvard, fue muy popular durante muchos años y se sigue utilizando en muchas revistas. Su gran ventaja es la comodidad para el autor. Como las referencias no están numeradas, pueden añadirse o suprimirse fácilmente.

Sistema numérico-alfabético. Este sistema consiste en citar por número de referencias de una lista alfabetizada, es una modificación moderna del sistema nombre y año. La cita por números mantiene los gastos de impresión dentro de límites razonables; la lista alfabetizada, especialmente si es larga, resulta relativamente fácil de preparar para los autores y de utilizar para los lectores.

Sistema de orden de mención. El sistema de orden de mención consiste sencillamente en citar las referencias (por número) según el orden en que se mencionan en el artículo. A los lectores con frecuencia este modo de proceder les gusta porque pueden acudir rápidamente a la lista de referencias si lo desean, siguiendo el orden numérico a medida que las encuentran en el texto. Es un sistema útil para las revistas en que cada artículo contiene sólo algunas referencias.

En la figura 5 veremos algunos ejemplos de cómo enunciar la literatura citada. A continuación algunas sugerencias:

Para la mención de revistas se indica el siguiente orden:

- Nombre de los autores, hasta un máximo de seis, separados por comas, con su apellido y las iniciales del nombre sin puntos (excepto tras la última inicial del último autor). Si sobrepasa el número de seis, hay que escribir los seis primeros y añadir "et al.", abreviatura de la expresión latina "et alii" que significa "y otros". Si el autor es un comité, se debe poner el nombre del comité.

- Título del trabajo, terminado con un punto.

- Revista biomédica, en su expresión abreviada según aparece en el index medicus, a la que sigue, sin puntuación alguna inmediata. 


\begin{tabular}{|c|c|}
\hline Sistema de nombre y año & $\begin{array}{l}\text { Day RA. 1996. Cómo escribir y publicar trabajos científicos. Washington, DC: Organi- } \\
\text { zación Panamericana de la Salud. } \\
\text { Huth EJ. 1986. Guidelines on authorship of medical papers. Ann Intern Med 104: } \\
269-274 \text {. } \\
\text { Sproul J, Klaaren H, Mannarino F. 1993. Surgical treatment of Freiberg's infraction in } \\
\text { athletes. Am J Sports Med 21: 381-384. }\end{array}$ \\
\hline Sistema numérico-alfabético & $\begin{array}{l}\text { 1. Day RA. 1996. Cómo escribir y publicar trabajos científicos. Washington, DC: } \\
\text { Organización Panamericana de la Salud. } \\
\text { 2. Huth EJ. 1986. Guidelines on authorship of medical papers. Ann Intern Med } \\
\text { 104: } 269-274 \text {. } \\
\text { 3. Sproul J, Klaaren H, Mannarino F. 1993. Surgical treatment of Freiberg's infraction } \\
\text { in athletes. Am J Sports Med 21: 381-384. }\end{array}$ \\
\hline Sistema de orden de mención & $\begin{array}{l}\text { 1. Huth EJ. Guidelines on authorship of medical papers. Ann Intern Med 1986; } \\
\text { 104:269-274. } \\
\text { 2. Sproul J, Klaaren H, Mannarino F. Surgical treatment of Freiberg's infraction in } \\
\text { athletes. Am J Sports Med 1993; 21:381-384. } \\
\text { 3. Day RA. Cómo escribir y publicar trabajos científicos. Washington, DC: Organización } \\
\text { Panamericana de la Salud; } 1996 .\end{array}$ \\
\hline
\end{tabular}

Figura 5. Algunos ejemplos de cómo enunciar la literatura citada, de acuerdo a los sistemas de nombre y vía numéricoalfabético y por orden alfabético.

- Año de publicación, -punto y coma-, volumen, - abrir paréntesis-, número o mes del ejemplar (esto puede omitirse si la paginación del volumen es consecutiva), -cerrar paréntesis-, -dos puntos-, página del artículo la primera y la última, si bien la última página puede indicarse con sólo el último dígito si los primeros fueran iguales a los de la primera página.

- Si se trata de capítulos de libros en los que varios autores han colaborado, se cita de la siguiente forma:

- Autor(es) del capítulo.

- Título del capítulo.

- Autor(es) del libro (denominados "editores", o título del libro).

- Ciudad donde se ha impreso.

- Editorial que lo ha publicado.

- Año de publicación.

- Páginas (primera y última) del capítulo.

Un aspecto importante de considerar es que las referencias se citaran según la normativa exigida por la revista elegida. Salvo casos de publicaciones de gran relevancia histórica, las citas deben ser recientes, donde al menos el $70 \%$ de ellas no pueden superar a los 5 años en caso de una revisión.

\section{Recomendaciones para mejorar el estilo de redacción}

Es necesario poner atención en la redacción a la construcción de párrafos que deben ser unidades de ideas y no de extensión. Se deben vincular párrafos para obtener una línea coherente de argumentos. También en la redacción se deben evitar descripciones poco concisas, exceso de retórica en la escritura, pero sobre todo el proclamar conclusiones no fundamentadas. Se apuntarán a continuación algunos de los vicios gramaticales más frecuentes:

- Utilizar mayúsculas para designar conceptos o palabras venerables, esto es lícito para literatura panfletaria no para proyectos de investigación (puede resaltarse un concepto con negrita o cursiva). Las mayúsculas se utilizan sólo para referirse personas física o morales.

- Incluir en una sola frase muchos interrogantes.

- Es conveniente consultar el diccionario para utilizar correctamente cada término.

- Un vicio galicista es abusar de expresiones en infinitivo. Por ejemplo: cuestión a plantear, debe decirse cuestión que se debe plantear, o la cuestión debatible. 


\section{Trucos para mejorar el lenguaje:}

- Es recomendable no abusar del alargamiento de palabras. Por ejemplo normativa, en vez de norma; totalidad, en reemplazo de todo; ejercer influencia, en vez de influir; recepcionar en vez de recibir, etc.

- Sin embargo, no todos los alargamientos son caprichosos, a veces la lógica del lenguaje científico exige la conversión de un sustantivo en un abstracto. Así por ejemplo el sustantivo religión, puede ser transformado en algo graduable, por lo tanto medible, y se convierte en religiosidad.

- No anunciar lo que se va a decir, hay que empezar directamente. Los enunciados o comentarios de cuadros no deben llevar la antesala retórica de "podemos afirmar", y pero aún, "estamos en condiciones de afirmar". Esto es común en discursos políticos, que necesitan decir el mayor número de palabras con el menor número posible de ideas.

- No abusar del prefijo "pre": preaviso, precalentamiento, pre-proyecto.

- Evitar adjetivos expresados como comparación, por ejemplo: "más evidente", "más objetivo", es similar a decir "más bueno".

- No componer oraciones con más de 30 palabras entre punto y punto.

- No abusar del verbo en participio pasado.

- Frase en forma pasiva: "la enfermedad es así percibida como ocasionada por un rasgo peligroso o por la acumulación de actos peligroso cometidos por un individuo enfermo" (23 palabras). Frase corregida: "se percibe la enfermedad como consecuencia del carácter o conducta arriesgada de los individuos" (16 palabras - se redujo tres casos de participio pasado).

- No confundir el significado de ciertas parejas de palabras que se parecen pero que tienen diferentes significado, ejemplos: transpolar (pasa por el polo) y extrapolar (proyectar la variable), revindicar (defender a un injuriado) y reivindicar (reclamar); mortalidad (fallecidos por habitante) y mortandad (cantidad extraordinaria de muertes).

- Huir de las palabras comodín tales como el adjetivo "importante": por ejemplo: "es importante empezar diciendo"

- Evitar reiteraciones enfáticas (figura 6).
Finalmente, el uso de los tiempos verbales en las secciones se resumen en:

- Resumen: pasado.

- Introducción: presente.

- Materiales y métodos: pasado.

- Resultados: pasado.

- Discusión: presente.

\section{Conclusiones}

Secreto 1: Parecer profesionales. Es importante mantener consistencia en el tamaño de la fuente (letras), en los márgenes, en los espacios entre líneas y en los tamaños y formatos de tablas y figuras. Todo debe ser nítido $\mathrm{y}$ claro.

Secreto 2: Escoger bien el mensaje. Todos los elementos del artículo deben apuntar al mensaje central y el mensaje central deriva de los datos. De modo que se deben mirar los datos y decidir que historia se va a contar, que es lo nuevo y que se ha demostrado. Luego contar la historia con figuras y tablas gastando horas y días jugando con ellas. Meta: el mensaje principal debe estar representado con tablas $\mathrm{y}$ figuras.

Secreto 3: Ponerse en el lugar del lector. El primer lector es: el revisor y luego el editor. La mayor parte de los revisores y editores, primero leen el título y una mirada de reojo a los autores. Luego leen la conclusión del resumen o el resumen entero. La mayor parte de los

\begin{tabular}{|c|c|}
\hline Reiteración enfática & Substituto o \\
\hline - Vuelvo a reiterar & - Reitero \\
\hline - Reafirmar tajantemente & - Reafirmar \\
\hline - Autoridad legítima & $\begin{array}{l}\text { - (Toda autoridad es } \\
\text { legítima) }\end{array}$ \\
\hline - Protagonismo principal & $\begin{array}{l}\text { - (No hay protagonistas } \\
\text { secundarios) }\end{array}$ \\
\hline - Prever de antemano & $\begin{array}{l}\text { - } \text { (Prever equivale a } \\
\text { antemano) }\end{array}$ \\
\hline - Proyecto de futuro & $\begin{array}{l}\text { - Los proyectos miran } \\
\text { siempre hacia el futuro }\end{array}$ \\
\hline - Bifurcar en dos direcciones & - ("Bi es dos") \\
\hline - Azar imprevisto & - (Todo azar es imprevisto) \\
\hline
\end{tabular}

Figura 6. Algunas reiteraciones enfáticas y los sustitutos adecuados o fundamento. 


\author{
Comprobación de errores \\ ¿Se envía a la revista adecuada? \\ ¿Se cumplen todas las instrucciones que el editor de la \\ revista indica? \\ ¿Son los autores los que realmente merecen el crédito del \\ trabajo? \\ ¿Es el resumen demasiado largo o demasiado complejo? \\ ¿Está correctamente estructurado el núcleo del trabajo: \\ IMRYD? \\ ¿Es la bibliografía reciente y citada según normativa propia \\ de la revista? \\ ¿Se corresponden las citas bibliográficas con lugar del texto \\ donde se citan? \\ ¿Se responde en la Conclusión a la pregunta formulada en \\ la Introducción? \\ ¿Son las tablas o figuras fácilmente inteligibles y resumen los \\ resultados? \\ ¿Están la Introducción y las Conclusiones escritas en presen- \\ te, y el Material y Resultados en pasado?
}

Figura 7. Lista conceptual de comprobación de errores.

buenos revisores, inmediatamente pasa a las figuras y tablas. De modo que se pierden muchas aceptaciones y por tanto lectores, por malos títulos, malos resúmenes y falta de figuras y tablas.

Por último, a riesgo de omitir puntos importantes, se deben chequear todos los puntos anteriores con el fin de corregir errores (figura
7). Las consideraciones expuestas en este artículo sólo constituyen una guía para profesionales del área biomédica, y sólo el ejercicio repetitivo de escribir artículos científicos, constituirá la base central y experiencia que cada profesional necesita.

\section{Referencias}

1.- Tuchman $B W$ : The book. Conferencia organizada por el Centro del Libro de la Biblioteca del Congreso y la Liga de Autores de los Estados Unidos. Biblioteca del Congreso, Washington, DC; 1980.

2.- Day RA: Cómo escribir y publicar trabajos científicos. 3a. Ed. Washington, Organización Panamericana de la Salud; 2005.

3.- Merton RK: La sociología de la ciencia. Alianza editorial Madrid; 1985.

4.- UNESCO: Guía para la redacción de artículos científicos destinados a la publicación. 2 ed. París UNESCO; 1983.

5.- Alonso-Soler M, Nereyda Piñeiro-Suárez N: ¿Cómo escribir un artículo científico? Alcmeon, Revista Argentina de Clínica Neuropsiquiátrica 2007; 16: 76-81.

6.- Russell de Galina J: El artículo científico. Conferencia U.N.A., Heredia; 1985.

7.- Ferriols R, Ferriols F: Escribir y publicar un artículo científico original. Ediciones Mayo, S.A. Aribau, 1857/08021 Barcelona Segre, 29 / 28002 Madrid; 2005.

8.- De la Torre R: Cómo presentar el capítulo de material y métodos. Conferencia U.N.A.M. México; 1984.

9.- Tobar F: Cómo redactar trabajos científicos en salud pública. http://www.isalud.org/htm/pdf/como-redactartrabajos-en-salud-publica.pdf. 